\title{
Automatic Detection of Alpine Ski Turns in Sensor Data
}

\author{
Michael Jones \\ Dept. of Computer Science \\ Brigham Young U. \\ Provo, UT 84602, USA \\ mike.jones@byu.edu \\ Zann Anderson \\ Dept. of Computer Science \\ Brigham Young $U$. \\ Provo, UT 84602, USA \\ zann@byu.net
}

\section{Casey Walker}

Dept. of Computer Science

Brigham Young U.

Provo, UT 84602, USA

\author{
Lawrence Thatcher \\ Dept. of Computer Science \\ Brigham Young U. \\ Provo, UT 84602, USA
}

\begin{abstract}
We experiment with using sensors and a machine learning algorithm to detect and label turns in alpine skiing. Previous work in this area involves data from more sensors and turns are detected using either a physics-based model or custom signal processing algorithm. We recorded accelerometer and gyroscope data using a single sensor placed on a skier's knee. Left and right turns in the data were labeled for use in machine learner. Although skiing data proved to be difficult to label precisely, a classifier trained on 37 labelled examples correctly label all 13 examples from a different test data set with 2 false positives. This method allows for the use of a single sensor and may be generalizable to other applications.
\end{abstract}

\section{Author Keywords}

Ubiquitous computing; Alpine skiing; Machine learning

\section{ACM Classification Keywords}

H.5.2 [Information interfaces and presentation (e.g., $\mathrm{HCl}$ )]: User Interfaces - User-centered design; I.5.4 [Pattern Recognition]: Applications - Signal processing

Ubicomp/ISWC'16 Adjunct, September 12-16, 2016. Heidelberg, Germany.

Copyright @ 2016 ACM ISBN978-1-4503-4462-3/16/09 \$15.00.

http://dx.doi.org/10.1145/2968219.2968535

\section{Introduction}

The availability of low-power and small but accurate sensors allows for applications wherein data from many different activities can be gathered and analyzed. Machine learn- 
ing provides a useful tool for understanding and analyzing data gathered in this way. Using this analysis, an event recognizer can be constructed and used as the foundation for an interactive system which tracks, responds to, or provides feedback about the activity. Mountains and mountain sports provide a rich and interesting area in which to apply these ideas.

There are several challenges involved in building real-time event recognizers for use in the mountains. These include environmental challenges often encountered in mountain environments such as cold, wind, snow, or exposure, and technical challenges such as where to mount the sensor, determining what data to collect, and determining how to recognize events in the sensor data stream.

We explore these challenges in the context of a system for recognizing alpine ski turns from data collected by a single sensor mounted on the skier's leg as shown in Figure 1. A ski turn recognizer could be used to build an interactive coaching system to be used while skiing or a "turn counter" for measuring exertion in a manner similar to a step counter. The coaching system might offer spoken feedback through an ear piece during skiing. The system might also be paired with a GPS system to provide location-aware feedback about style or performance.

Others have approached the specific problem of detecting and understanding turns in alpine snow sports. Adelsberger [1] used 30 sensors and a physics based model to measure ski deflection during turns in alpine skiing. Spelmezan [5] used 8 sensors and a signal processing algorithm to detect snowboarding turns. We use a single sensor and apply a machine learning algorithm to learn a classifier from labeled training data. Learning a classifier from examples may remove the need for special expertise in building a custom

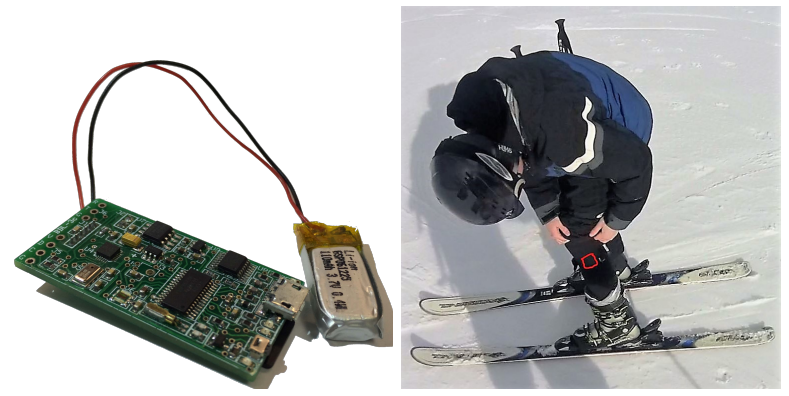

Figure 1: Left: The sensor used to collect acceleration and rotation data together with a small $110 \mathrm{mAh}$ battery. Right: placement of the sensor on the skier's left leg just below the knee as marked by the red box.

model or algorithm, and may be generalizable to other applications.

The sensor records acceleration and rotation in three dimensions each while a skier makes turns. Left and right turns are labeled in the data and the labeled data is given to a machine learning algorithm based on automated speech recognition (ASR). We trained a classifier on 37 labeled examples and tested on a different data set containing 13 labeled examples. The classifier correctly identified all 13 turns and had 2 false positives in which it incorrectly labeled data as being part of a turn.

\section{Collecting Data}

Data is collected by attaching the sensor to a skier and recording data about the skier's movements. The sensor is shown in the left side of Figure 1. The sensor is a PCB that contains an accelerometer and gyroscope along with a processor and SD card slot. The board together with a small $110 \mathrm{mAh}$ battery measure $47 \times 23 \times 9 \mathrm{~mm}$. 
The sensor is designed to be small and lightweight to simplify deployment in different applications and environments, and can run for 1 hour and 15 minutes using the battery. The sensor records acceleration and rotation on three axes at a rate of 80 samples per instrument per second. The data are written to an SD card to be processed later. We enclosed the sensor and battery in a waterproof housing. A cap on the housing allows easy access to the sensor's on-off switch.

We initially placed the sensor on the ski but moved the sensor to the skier's leg below the knee as shown on the right side of Figure 1. A red box in the figure highlights the sensors placement. When placed on the ski halfway between the boot and the ski tip, the sensor recorded nearly constant noise from ski vibration when the skier was in motion. This noise masked information that could have been used to recognize a turn in the sensor data. Placing the sensor below the knee dampens vibration while capturing the angle of the lower leg and, by extension, the angle of the ski itself. We did not explore other placements on the skier, such as on the back or helmet. Other placements may provide better data and may further reduce the risk of an injury in case of a crash or fall.

It also proved difficult to turn the sensor on and off when mounted on the ski because the user could not reach the on-off switch without taking a ski off. Placing the sensor below the knee puts the sensor in the skier's reach for easy access to the on-off switch. The switch in this design is very small and difficult to operate while wearing a glove or mitten. Future iterations of the design will need to consider operation while wearing a glove.

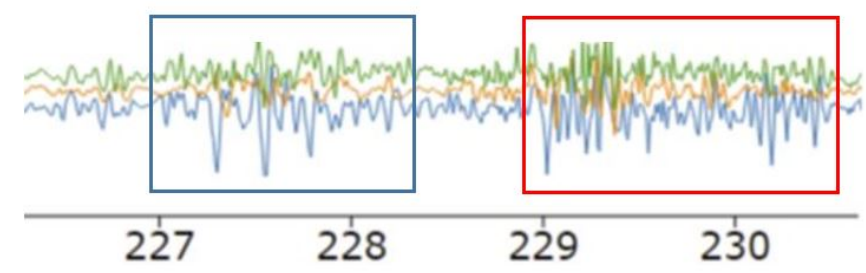

Figure 2: Acceleration data collected from a sensor mounted to a skier's left leg just below the knee. Time in seconds is shown along the bottom axis. The blue box encloses a turn to the left and the red box encloses a longer turn to the right.

\section{Labeling Data}

For the alpine skiing data, we chose to label two events: Turn Left and Turn Right, with each event being subdivided into a first and a second half. Each turn begins when the skier begins movement in the direction of the turn. The first half of the turn ends when the skier reaches the apex of the turn. The turn ends when the skier begins moving along a straight line at the end of the turn. A sample of two labeled turns is shown in Figure 2. We collected video along with the sensor data and used the video to aid in labelling the data.

The locations of the event boundaries proved hard to determine from the data, however the labeling which was done on training data allowed the learner to be quite accurate in labeling events in test data.

\section{Learning a Classifier}

The machine learning algorithm takes labeled data as input and learns a classifier for identifying ski turns in sensor data streams. The algorithm is based on the observation that recognizing fragments of events in sensor data streams is similar to recognizing fragments of spoken words in au- 
dio data streams. Based on that idea, we adapted the ASR process found in [2] for training a Gaussian-mixture model based hidden Markov model from labeled training data. Our implementation is based on an implementation of hidden Markov models described in [6]. The Gaussian mixture model and the hidden Markov model have been used by others to recognize events in streaming sensor data [3, 4].

The training data and the testing data were collected from a single skier on two different days. Training data were collected on a day with $1 \mathrm{~cm}$ of loose snow on top of a hard packed snow base. Testing data were collected on a day with no loose snow and a hard packed base. The training data included 37 parallel turns made in a single run with 19 right turns and 18 left turns.

We measured the correctness of the classifier using an event-based metric with a tolerance factor. In this metric, labels created by the classifier are considered correct if they overlap in time the manually-labelled event in any way. For example, we say that the classifier is correct even if it labels a right turn event starting a second before the labelled event and ending during the event. Although the inferred label does not match the actual label, the overlap indicates that the algorithm correctly identified a turn.

The rationale for this approach is that we are interested, for now, in recognizing turns rather than recognizing the instant in which a turn began or ended. Obviously, in some applications the precise beginning and ending of a turn is important. Our correctness metric is not suitable for measuring performance in those types of applications. We hypothesize that the classifier fails to detect the exact start and stop points for a turn because there is significant variation in each turn. Different snow conditions, slope angles, and turn radii lead to differences in data collected from turn to turn and this may lead to inaccuracies in classification.
The classifier correctly labeled all 13 turns in a test data set with 7 left turns and 6 right turns. However, in two cases the classifier incorrectly added a "right turn" label to a left turn (in addition to correctly labeling that turn as a left turn).

While we tested the classifier on a pre-recorded data set, the aim is to run the classifier on streaming data as the skier makes turns. This allows the classifier to detect turns in real time.

\section{Discussion}

Training a classifier to recognize left and right alpine skiing turns from a set of labeled examples was surprisingly effective. The difference in ski conditions on the day we gathered test data almost certainly impacted our performance as did the fact that we had only 37 turns of data to learn from. In addition, labeling turns in the data proved difficult because alpine ski turns involve fluid motion and it can be difficult to identify where a turn begins and ends. Nevertheless, our somewhat imprecise labels resulted in a reasonably accurate classifier and did so based on learning from the labeled data, rather than a detailed study of the mechanics of skiing motion or the generated data stream.

We used only one sensor, which proved adequate for recognizing the occurrence and direction of a single kind of alpine ski turn. More sensors may generate a richer data stream to support more nuanced and precise classification of turn parameters such as quality or type. Using additional sensors, however, comes at the cost of requiring the user to wear, start, and manage more electronics while skiing.

We tested placement on the skier's knee and ski. Other placements may result in data that is better suited for classification or allow for classification of different events. For example, placing a sensor on a ski pole might allow for coaching a skier on pole placement during a turn. 


\section{REFERENCES}

1. Rolf Adelsberger, Sämy Aufdenblatten, Matthias Gilgien, and Gerhard Tröster. 2014. On bending characteristics of skis in use. Procedia Engineering 72 (2014), 362-367.

2. D. Jurafsky and J.H. Martin. 2009. Speech and Language Processing: An Introduction to Natural Language Processing, Computational Linguistics, and Speech Recognition. Pearson Prentice Hall.

3. Donald J Patterson, Dieter Fox, Henry Kautz, and Matthai Philipose. 2005. Fine-grained activity recognition by aggregating abstract object usage. In Wearable Computers, 2005. Proceedings. Ninth IEEE International Symposium on. IEEE, 44-51.
4. Thomas Plötz, Paula Moynihan, Cuong Pham, and Patrick Olivier. 2011. Activity recognition and healthier food preparation. In Activity Recognition in Pervasive Intelligent Environments. Springer, 313-329.

5. Daniel Spelmezan. 2012. An investigation into the use of tactile instructions in snowboarding. In Proceedings of the 14th international conference on

Human-computer interaction with mobile devices and services. ACM, 417-426.

6. Steve Young, Gunnar Evermann, Mark Gales, Thomas Hain, Dan Kershaw, Xunying Liu, Gareth Moore, Julian Odell, Dave Ollason, Dan Povey, and others. 1997. The HTK book. Vol. 2. Entropic Cambridge Research Laboratory Cambridge. 